\title{
Porosity models for pre-planetesimals: modified $P-\alpha$ like models and the effect of dissipated energy
}

\author{
Serena E. Arena ${ }^{1}$ and Roland Speith ${ }^{2}$ \\ ${ }^{1}$ Université de Lyon, Lyon, F-69003, France; Université Lyon 1, Villeurbanne, F-69622, France; \\ CNRS, UMR 5574, Centre de Recherche Astrophysique de Lyon; \\ École normale supérieure de Lyon, 46, allée d'Italie, F-69364 Lyon cedex 07, France \\ email: serena.arena@ens-lyon.fr \\ ${ }^{2}$ Physikalisches Institut, Universität Tübingen, Auf der Morgenstelle 14, D-72076 Tübingen, \\ Germany \\ email: speith@pit.physik.uni-tuebingen.de
}

\begin{abstract}
The outcome of collisions between pre-planetesimals is important in the theory of planetesimal formation by collisional growth and strongly depends on their internal structure. Since pre-planetesimals are highly porous, reaching $90 \%$ porosity, they could show the so called anomalous behaviour (decrease of density during shock compression, e.g. Bolkhovitinov \& Khvostov 1978). Due to involved sizes ( $>\mathrm{dm}$ ), laboratory experiments are unfeasible therefore numerical simulations equipped with adequate porosity models are necessary.

Here we focus on the $P-\alpha$ model and its variations. We found that they are suitable for applications in the high porosity range only after a modification of the basic equations, that avoids an inconsistency and takes into account the effect of dissipated energy, is performed.
\end{abstract}

Keywords. equation of state, shock waves, interplanetary medium

In the $P$ - $\alpha$ model (Herrmann 1969; Carroll \& Holt 1972) the equation of state (EOS) of the porous material is derived from that of its solid matrix (subscript $m$ ) by the introduction of the distension parameter $\alpha=\rho_{m} / \rho$, relating the respective density, and the assumption that the specific internal energy of the two components is the same $U=U_{m}$. The $P-\alpha$ model and its modified $\varepsilon-\alpha$ variation have been respectively used by Jutzi et al. (2008) and Wünnemann et al. (2006) in the related field of impact cratering but only for low and intermediate porosity up to $60 \%$. However at high porosity we found that the first is affected by a singularity and the second cannot model anomalous behaviour of the porous material.

The inconsistent $U=U_{m}$ assumption.

This assumption implies $\mathrm{d} U=\mathrm{d} U_{m}$, but this is valid only in the unrealistic situation where the material maintains a constant porosity $(\alpha=$ const) while it is subject to compression. In fact, applying the First Principle at both the porous material and its matrix we have: $\mathrm{d} U=\mathrm{d} U_{m}-\frac{P_{m}}{\alpha \rho_{m}} \mathrm{~d} \alpha$, where the definition of distension $\alpha$ is used and $\delta Q=\delta Q_{m}$ because the pores in the porous material are assumed to be empty.

The suggested modification.

In order to avoid the singularity, we suggest to derive the internal energy of the matrix directly from the First Principle, substituting $U_{m}=U$ with $\mathrm{d} U_{m}=\frac{P_{m}}{\rho_{m}^{2}} \mathrm{~d} \rho_{m}+\delta Q_{m}$. The term $\delta Q_{m}$ represents the heat exchanged by the matrix with its environment and for high porous materials it is mainly due to the energy dissipated during plastic deformation.

Application: shock compression.

We use the Hugoniot as energy equation and the simplified Tillotson as matrix EOS. 

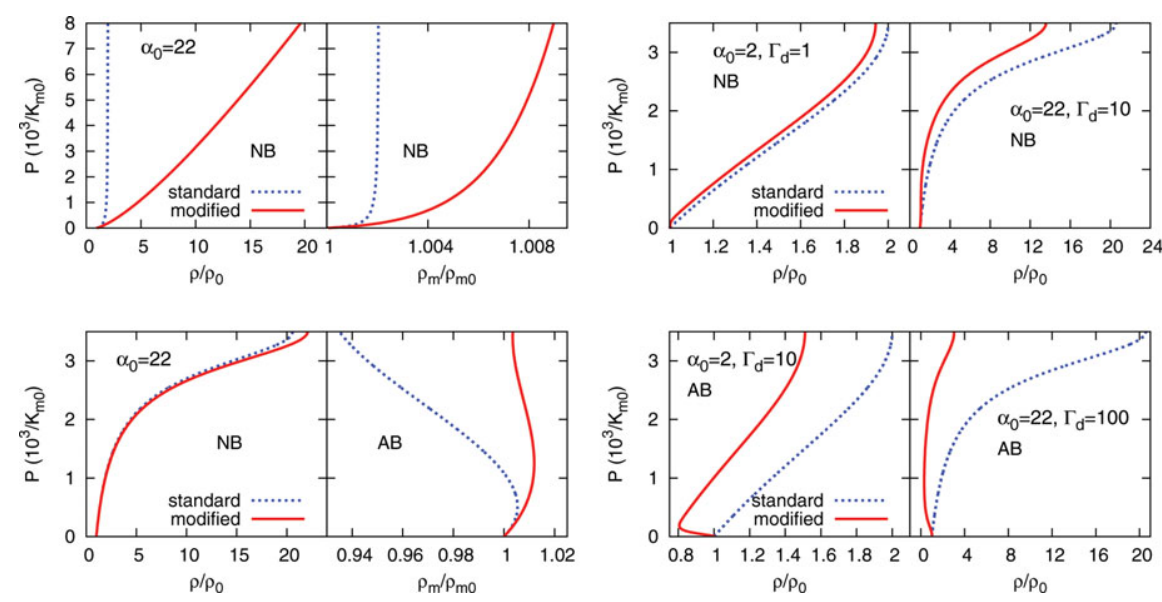

Figure 1. Left: standard (short dashed line) and modified (solid line) $\varepsilon-\alpha$ models (top panel) and $P$ - $\alpha$ models (bottom panel) without energy dissipation for high porosity materials. Right: standard and modified $P-\alpha$ models with energy dissipation for a low porosity material (left plot in each panel) and high porosity material (right plot in each panel).

The modified $P-\alpha$ model without dissipation. The singularity, located at $\rho_{\text {sing }} / \rho_{0}=2$ in the standard $\varepsilon$ - $\alpha$ model (short dashed line in the top left panel in the figure), disappears in the modified model (solid line in the same panel). This model predicts normal behaviour (NB) for both the porous material and its matrix. The bottom left panel shows that both in the standard and in the modified $P-\alpha$ model the behaviour of the porous material is normal, even if the density of the matrix presents a slight anomalous behaviour.

The effects of dissipated energy. The heat exchange is written as: $\Delta Q_{m}=\Gamma_{d} \int_{\alpha_{0}}^{\alpha} M\left(\alpha^{\prime}\right) \mathrm{d} \alpha^{\prime}$, where the normalised local dissipation function $M(\alpha)$ is taken to peak at high porosity and the dissipation coefficient $\Gamma_{d}$ regulates the magnitude of the dissipation. In the modified version of the $P-\alpha$ model a critical value $\Gamma_{d_{C}}$ exists for each configuration such that below it the model predicts normal behaviour for the porous material independently of the initial porosity (top right panel in the figure) and above it anomalous behaviour is predicted for the porous material independently of the initial porosity (bottom right panel in the figure). For modified $\varepsilon$ - $\alpha$ models only normal behaviour can be reproduced and the dissipated energy has the only quantitative effect of moving the state of full compaction toward higher pressure.

Conclusions. The correction of the inconsistency resolves the singularity problem and the inclusion of dissipated energy allows the description of both normal and anomalous behaviour. Standard $P$ - $\alpha$ like models can be extended to the high porosity range, however the expected features of pre-planetesimals can be better described by the $P-\alpha$ modified model because the $\varepsilon-\alpha$ model is restricted only to normal behaviour.

Acknowledgements. SEA acknowledges funding by the German Science Foundation DFG under grant SP 646/1-1.

\section{References}

Bolkhovitinov, L. G. \& Khvostov, Y. B., 1978, Nature, 274, 882

Herrmann, W. 1969, Journal of Applied Physics, 40, 2490

Carroll, M. \& Holt, A. C., 1972, Journal of Applied Physics, 43, 759

Jutzi, M., Benz, W., \& Michel, P., 2008, Icarus, 198, 242

Wünnemann, K., Collins, G. S., \& Melosh, H. J., 2006, Icarus, 180, 514 